
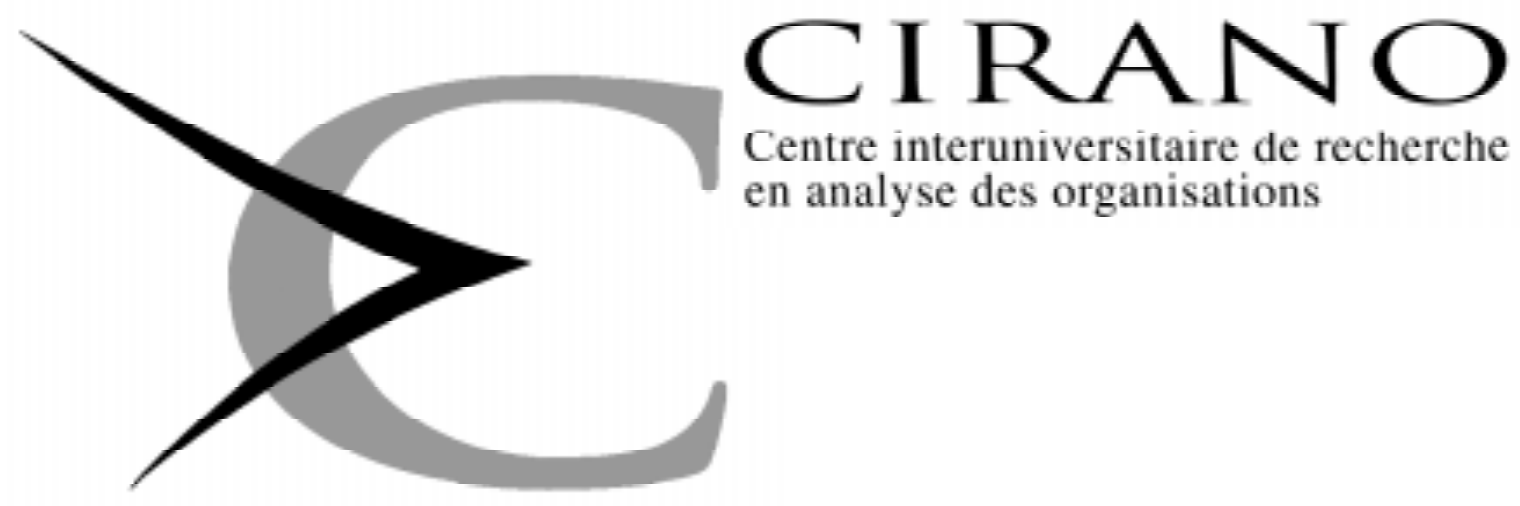

Centre interuniversitaire de recherche en analyse des organisations

Série Scientifique

Scientific Series

No 94s-11

SIMULATION BASED

INFERENCE IN MOVING

AVERAGE MODELS

Eric Ghysels, Linda Khalaf,

Cosmé Vodounou 


\section{CIRANO}

Le CIRANO est une corporation privée à but non lucratif constituée en vertu de la Loi des compagnies du Québec. Le financement de son infrastructure et de ses activités de recherche provient des cotisations de ses organisations-membres, d'une subvention d'infrastructure du ministère de l'Industrie, du Commerce, de la Science et de la Technologie, de même que des subventions et mandats obtenus par ses équipes de recherche. La Série Scientifique est la réalisation d'une des missions que s'est données le CIRANO, soit de développer l'analyse scientifique des organisations et des comportements stratégiques.

CIRANO is a private non-profit organization incorporated under the Québec Companies Act. Its infrastructure and research activities are funded through fees paid by member organizations, an infrastructure grant from the Ministere de l'Industrie, du Commerce, de la Science et de la Technologie, and grants and research mandates obtained by its research teams. The Scientific Series fulfils one of the missions of CIRANO: to develop the scientific analysis of organizations and strategic behaviour.

\section{Les organisations-partenaires / The Partner Organizations}

-Ministère de l'Industrie, du Commerce, de la Science et de la Technologie. -École des Hautes Études Commerciales.

-École Polytechnique.

- Université de Montréal.

-Université Laval.

-McGill University.

-Université du Québec à Montréal.

-Bell Québec.

-Caisse de dépôt et de placement du Québec.

-Hydro-Québec.

-Banque Laurentienne du Canada.

-Fédération des caisses populaires de Montréal et de l'Ouest-du-Québec.

Ce document est publié dans l'intention de rendre accessible les résultats préliminaires de la recherche effectuée au CIRANO, afin de susciter des échanges et des suggestions. Les idées et les opinions émises sont sous l'unique responsabilité des auteurs, et ne représentent pas nécessairement les positions du CIRANO ou de ses partenaires.

This paper presents preliminary research carried out at CIRANO and aims to encourage discussion and comment. The observations and viewpoints expressed are the sole responsibility of the authors. They do not necessarily represent positions of CIRANO or its partners.

ISSN 1198-8177 


\title{
Simulation Based Inference in Moving Average Models
}

\author{
Eric Ghysels ${ }^{\dagger}$ \\ Linda Khalaf \\ and \\ Cosmé Vodounou ${ }^{\ddagger}$
}

\begin{abstract}
Résumé
We examine several simulation-based estimators for the parameters of a moving average process, including the one initially proposed by Gouriéroux, Monfort and Renault (1993) as well as several extensions based on Gallant and Tauchen (1994). The estimators are also compared and related to procedures recently suggested by Galbraith and Zinde-Walsh (1994).

Nous examinons plusieurs estimateurs basés sur les principes des méthodes de moments simulés et l'inférence indirecte pour des modèles de moyenne mobile. Nous étudions une procédure proposée par Gouriéroux, Monfort et Renault (1993) ainsi que des extensions de l'approche proposée par Gallant et Tauchen (1994). Nous faisons également une comparaison avec les procédures de Galbraith et Zinde-Walsh (1994).
\end{abstract}

JEL Classification: C22, C20, C32

Correspondence: Eric Ghysels, C.R.D.E., Université de Montréal C.P. 6128, Succ. centre-ville, Montréal (Québec) Canada, H3C 3J7 Fax: (514) 343-5831

E-mail: eghysels@plgcn.umontreal.ca

$\dagger$ C.R.D.E., Université de Montréal and CIRANO

$\ddagger$ C.R.D.E., Université de Montréal 


\section{Introduction}

There are several estimation procedures for moving average models. A large class of estimators are likelihood-based and involve (numerical) optimization of the exact or approximate likelihood function ${ }^{1}$. Another class relies either on the estimation of a sequence of long autoregressions, as in Hannan and Rissanen (1982) and Koreisha and Pukkila (1990) or else involves nonlinear least squares (see, for instance, Fuller (1976) for a discussion). Durbin (1959) suggested fitting an autoregression of finite order to the data and deriving an estimate for the MA parameter ${ }^{2}$. Recently, Galbraith and Zinde-Walsh (1994) proposed a related estimator which involves approximating, in the Hilbert Norm, a MA model by an autoregressive (AR) model. It uses a minimal Hilbert distance criterion to describe explicitly a functional form between the MA parameters and the "auxiliary" AR parameters.

In this paper, we propose several simulated method of moments (SMM) estimators for the parameters of a Gaussian MA(1) process using a finite autoregression. Gouriéroux, Monfort and Renault (1993) provided a first example of such an estimator. The asymptotically equivalent procedure of Gallant and Tauchen (1993) is also considered. Hence we propose several alternatives SMM estimators and conduct a comparative study through Monte Carlo simulations. One advantage of SMM estimators is that, unlike the Durbin and Galbraith/Zinde-Walsh procedures, they do not require an explicit relation between the MA and AR parameters. Another advantage, stressed by Gouriéroux et al. (1993) is that simulation based method appear to correct in small samples the bias due the lag truncation in the AR representation. Moreover, we also reconsider the Galbraith/Zinde-Walsh $(\mathrm{G} / \mathrm{ZW})$ estimator and suggest an alternative asymptotic least squares (ALS) procedure that improves its large sample properties. In general, the discussion has focused on the performance of estimators near the boundary of the non-invertibility region where procedures like maximum likelihood estimation (MLE) appear to behave poorly in finite samples. Our Monte Carlo evidence shows that the SMM estimators perform better than Durbin and G/ZW's estimators for values of the MA parameter near unity.

\footnotetext{
${ }^{1}$ See for instance in Box and Jenkins (1976), Godolphin (1977), Osborn (1977), Ansley (1979) and Ansley and Newbold (1980).

${ }^{2}$ This relatively straightforward procedure has attracted considerable attention in the literature; see, for example, McClave (1973) or Mentz (1977) for a discussion of a statistical properties of Durbin's estimator.
} 
The paper is organized as follows. In section 2, we present our notational framework, introduce the different SMM and ALS estimators and discuss their asymptotic distributional properties. In section 3 , we report simulation results comparing the finite sample performance of the different estimators. Section 4 concludes.

\section{Notation and Proposed Estimators}

For convenience we shall devote our attention exclusively to the first order univariate MA model. Extensions to more general models will be discussed in Section 4. The idea to use simulation-based estimators for MA models as suggested in Gouriéroux et al. (1993) is further explored in several directions in this section. Three classes of estimators for the MA parameter will be considered. The first is based on a score principle as put forward by Gallant and Tauchen (1993). The second follows Gouriéroux et al. and can be characterized as a SMM estimator based on a Wald principle. Finally, we reconsider the G/ZW estimator and replace their OLS procedure by an ALS estimator ${ }^{3}$. We fix notation in section 2.1. Section 2.2 is devoted to the SMM estimators while 2.3 covers the ALS estimators. In section 2.4, we discuss the symptotic distributions of the proposed estimators.

\subsection{Notation and definitions}

We consider the time series model

$$
X_{t}=u_{t}-\theta u_{t-1}, t=1, \ldots, T,
$$

where $0<|\theta|<1$ and the $u_{t}$ are independent, identically distributed, normal random variables with $E u_{t}=0, E u_{t}^{2}=1$ for all t. The approximating AR(p) model will be denoted as

$$
X_{t}=\beta_{1} X_{t-1}+\cdots+\beta_{p} X_{t-p}+\varepsilon_{t}, t=1, \ldots, T,
$$

where $\varepsilon_{t}$ is a white noise. For a sample of size $T$, let

$$
\hat{\beta}_{(T)}^{p}=\left[\hat{\beta}, \ldots, \hat{\beta}_{p}\right]
$$

where $\hat{\beta}_{i}$ are the ordinary least squares estimates of $\beta_{i}, i=1, \ldots, p$, in the regression of $X_{t}$ on $X_{t-1}, \ldots, X_{t-p}$. The usual central limit theorem arguments yield that $\left(\hat{\beta}_{i}-\beta_{i}\right)$ is $O_{p}\left(T^{-\frac{1}{2}}\right)$ where

\footnotetext{
${ }^{3}$ See Gouriéroux, Monfort and Trognon (1985) for discussion.
} 


$$
\beta_{i}=-(-\theta)^{i}\left[\frac{1-\theta^{2(p-i+1)}}{1-\theta^{2(p+1)}}\right], i=1, \ldots, p^{4}
$$

\subsection{SMM estimators of the MA parameter}

For a given value of the MA in (2.1), we can obtain $H$ simulated paths

$$
\left[\tilde{X}_{1}^{h}(\theta), \ldots, \tilde{X}_{T}^{h}(\theta)\right], h=1, \ldots, H,
$$

based on independent drawings of $u_{t},\left[\tilde{u}_{1}^{h}, \ldots, \tilde{u}_{T}^{h}\right], h=1, \ldots, H$. For each of these paths, define

$$
\tilde{\beta}_{(T)}^{h p}(\theta)=\underset{\beta}{\operatorname{Argmin}} \tilde{Q}_{T, h}^{(p)}(\theta), h=1, \ldots, H,
$$

where

$$
\tilde{Q}_{T, h}^{(p)}(\theta)=\Sigma_{t=p+1}^{T}\left[\tilde{X}_{t}^{h}-\beta_{1} \tilde{X}_{t-1}^{h}-\ldots-\beta_{p} \tilde{X}_{t-p}^{h}\right]^{2}, h=1, \ldots, H .
$$

In other words, $\tilde{\beta}_{(T)}^{h p}$ minimizes the least squares criterion where the observed values are replaced by the simulated ones. Moreover, we define an estimator for $\beta$ as follows:

$$
\tilde{\beta}_{T H}^{p}(\theta)=\frac{1}{H} \sum_{h=1}^{H} \tilde{\beta}_{(T)}^{h p}(\theta) .
$$

Alternatively, we can obtain a sequence of $N=T H$ simulated values $\tilde{X}_{1}, \ldots, \tilde{X}_{N}$ based on $N$ drawings of the white noise process $u_{t}$, to which one would apply the following estimation criterion

$$
\tilde{Q}_{N}^{(p)}(\theta)=\Sigma_{t=p+1}^{N}\left[\tilde{X}_{t}-\beta_{1} \tilde{X}_{t-1}, \ldots, \beta_{p} \tilde{X}_{t-p}\right]^{2}
$$

Once again one can apply the criterion appearing in (2.6) and define $\beta_{N}^{p}(\theta)=\operatorname{Argmin} \tilde{Q}_{N}^{(p)}(\theta)$. We can now define two indirect estimators of $\theta$ as follows:

$$
\hat{\theta}_{T H p}^{S W}=\underset{\theta}{\operatorname{Argmin}}\left(\hat{\beta}_{(T)}^{p}-\hat{\beta}_{T H}^{p}(\theta)\right)^{\prime} \hat{\Omega}_{T}\left(\hat{\beta}_{(T)}^{p}-\hat{\beta}_{T H}^{p}(\theta)\right),
$$

\footnotetext{
${ }^{4}$ For later use, $\beta_{i}$ in (2.4) will be denoted as a function of $\theta$, namely $\beta_{i}=\beta_{i}(\theta)$.
} 
where $\hat{\beta}_{(T)}^{p}$ is defined as in (2.3) and $\tilde{\beta}_{T H}^{p}$ as in (2.8) while the weighting matrix is obtained as:

$$
\hat{\Omega}_{T}=\left[\left(\frac{\partial \hat{Q}_{T}^{(p)}}{\partial \beta}\right)\left(\frac{\partial \hat{Q}_{T}^{(p)}}{\partial \beta}\right)^{\prime}\right]^{-1},
$$

using the sample data to compute

$$
\hat{Q}_{T}^{(p)}=\sum_{t=p+1}^{T}\left(X_{t}-\hat{\beta}_{1} X_{t-1}-\cdots-\hat{\beta}_{p} X_{t-p}\right)^{2} .
$$

Replacing $\tilde{\beta}_{T H}^{p}(\theta)$ by $\tilde{\beta}_{N}^{p}(\theta)$ in section $(2.10)$ yields a second SMM estimator, namely

$$
\hat{\theta}_{N p}^{S W}=\underset{\theta}{\operatorname{Argmin}}\left(\hat{\beta}_{(T)}^{p}-\tilde{\beta}_{N}^{p}(\theta)\right)^{\prime} \hat{\Omega}_{T}\left(\hat{\beta}_{(T)}^{p}-\tilde{\beta}_{N}^{p}(\theta)\right) .
$$

Both estimators bear the superscript $S W$ indicating that they are simulation-based exploiting a Wald principle. Gouriéroux et al. (1993) show that the estimators defined in (2.10) and (2.13) have the same asymptotic distributions given appropriate regularity conditions. They also argue that in this particular application, the efficiency loss resulting from replacing $\hat{\Omega}_{T}$ by an identity matrix is marginal and therefore suggest to use the latter in practical applications. A third version of the indirect estimator, following Gallant and Tauchen (1993), can be formulated as follows:

$$
\hat{\theta}_{N p}^{S S}=\underset{\theta}{\operatorname{Argmin}}\left[\frac{\theta \tilde{Q}_{N}^{(p)}}{\partial \beta}(\theta)\right]^{\prime} \hat{\Omega}_{T}\left[\frac{\theta \tilde{Q}_{N}^{(p)}}{\partial \beta}(\theta)\right] .
$$

The advantage of this estimator is that it does not involve estimating $\beta$ from the simulated data as it relies on the score function, hence the superscript $S S$. A fourth estimator, denoted $\hat{\theta}_{T H p}^{S . S}$, can also be defined by combining arguments in (2.10) and (2.14).

\subsection{ALS estimators of the MA parameter}

The simulation-based estimators discussed in the previous section lead also to a class of estimators not involving simulation yet based on methods of moments principles. For instance, G/ZW proposed an estimator of $\theta$ based on $\hat{\beta}_{(T)}$ and the following approximate explicit relation inferred from the Yule-Walker equations: 


$$
\hat{\theta}_{G / Z W}=\hat{\beta}_{1} .
$$

They utilize the approximation (2.4) to evaluate the estimator's asymptotic bias. Obviously, this just identified estimator could be replaced by an ALS estimator exploiting the result in (2.4). Namely consider the estimator defined as

$$
\hat{\theta}_{p}^{A}=\underset{\theta}{\operatorname{Argmin}}\left(\hat{\beta}_{(T)}^{p}-\beta^{p}(\theta)\right)^{\prime} \hat{\Omega}_{T}\left(\hat{\beta}_{(T)}^{p}-\beta^{p}(\theta)\right),
$$

where $\beta^{p}(\theta)=\left(\beta_{1}(\theta), \ldots, \beta_{p}(\theta)\right)^{\prime}$ is defined in (2.4). Again, we also consider not using $\hat{\Omega}_{T}$ as a weighting matrix and rather rely on an asymptotically less efficient procedure. Both estimators have a standard asymptotic distribution as described in Gouriéroux, Monfort and Trognon (1985). One can readily see the direct relationship between the $\hat{\theta}_{p}^{A}$ and the simulationbased estimators $\hat{\theta}_{T H p}^{S W}$ and $\hat{\theta}_{N p}^{S W}$. The former relies on the asymptotic $O_{p}\left(T^{-\frac{1}{2}}\right)$ expansion typically used in the central limit theorem development of the OLS estimator. The latter two estimators replace the $\beta^{p}(\theta)$ expression by simulated or averages of simulated estimators. This difference is important to understand the finite sample behavior of the estimators.

\subsection{Asymptotic distributions of suggested estimators}

In this section we briefly summarize without any formal proof the asymptotic distributions of the estimators discussed in sections 2.2 and 2.3. To simplify the presentation, consider the following notation $\hat{\theta}_{j}^{i}\left(S_{T}\right)$, with $i=S W, S S$ and $A ; j=T H p, N p$ (for $S W$ and $S S$ ) and $j=p($ for $i=A$ ) while $S_{T}$ represents the weighting matrix satisfying

$$
S_{0}=\lim _{T \rightarrow \infty} S_{T}
$$

Moreover, let

$$
b^{p}(\theta)=\lim _{T \rightarrow \infty} \tilde{\beta}_{(T)}^{h p}(\theta),
$$

where $\tilde{\beta}_{(T)}^{h p}(\theta)$ is defined by (2.6). As (2.18) is for any arbitrary $h$ the index is dropped from the LHS of the equation. Furthermore, let $\Omega_{0}$ be the information matrix based on the score function considered. In the notation of Gouriéroux, Monfort and Renault (1993), the following special case corresponds to the problem on hand $K_{0}=0$ and $J_{0}=I_{0}=\Omega_{0}$. Moreover, it should be emphasized that $T^{\frac{1}{2}}\left(\hat{\beta}_{(T)}^{p}-\beta^{p}\left(\theta_{0}\right)\right)$ has $\Omega_{0}$ as an 
asymptotic covariance matrix, where $\beta^{p}(\theta)$ is given by $(2.16), \hat{\beta}_{(T)}^{p}$ by $(2.3)$ and $\theta_{0}$ is the true value of the MA parameter. We first examine the ALS estimator, namely

Proposition 3.1: The ALS estimator defined in (2.16) has, under suitable regularity conditions, the following asymptotic distribution:

$$
\begin{aligned}
& T^{\frac{1}{2}}\left(\hat{\theta}_{p}^{A}\left(S_{T}\right)-\theta_{0}\right) \stackrel{\stackrel{d}{\rightarrow}}{\rightarrow} N\left(0, \Sigma_{p}^{A}\left(S_{0}\right)\right) \text {, where } \\
& \Sigma_{p}^{A}\left(S_{0}\right)=\left[\frac{\partial \beta^{p}(\theta)^{\prime}}{\partial \theta} S_{0}^{-1} \frac{\partial \beta^{p}(\theta)^{\prime}}{\partial \theta}\right]^{-1} \frac{\partial \beta^{p}(\theta)^{\prime}}{\partial \theta} S_{0} \Omega_{0} S_{0} \frac{\partial \beta^{p}(\theta)}{\partial \theta}\left[\frac{\partial \beta^{p}(\theta)^{\prime}}{\partial \theta} S_{0}^{-1} \frac{\partial \beta^{p}(\theta)^{\prime}}{\partial \theta}\right]_{(2.19)}^{-1}
\end{aligned}
$$

The proof and regularity conditions appear in Gouriéroux et al. (1985). From (2.19) we also note that the optimal choice of $S_{0}$ is $\Omega_{0}^{-1}$. We examine the estimator $\hat{\theta}_{i}^{S W}$ namely

Proposition 3.2: Under suitable regularity conditions, $T^{\frac{1}{2}}\left(\hat{\theta}_{i}^{S W}\left(S_{T}\right)-\theta_{0}\right) \underset{T \rightarrow \infty}{\stackrel{d}{\rightarrow}} N\left(0, \Sigma_{p}^{S W}\left(S_{0}\right)\right)$, where $i=T H_{P}, N_{P}$, and

$$
\Sigma_{p}^{S W}\left(S_{0}\right)=\left(1+\frac{1}{H}\right)\left[\frac{\partial b^{p}(\theta)^{\prime}}{\partial \theta} S_{0}^{-1} \frac{\partial b^{p}(\theta)}{\partial \theta}\right]^{-1} \frac{\partial b^{p}(\theta)^{\prime}}{\partial \theta} S_{0} \Omega_{0} S_{0} \frac{\partial b^{p}(\theta)}{\partial \theta}\left[\frac{\partial b^{p}(\theta)^{\prime}}{\partial \theta} S_{0}^{-1} \frac{\partial b^{p}(\theta)^{\prime}}{\partial \theta}\right]_{(2.20)}^{-1}
$$

The proof and regularity conditions for this result appear in Gouriéroux et al. (1993). We notice two differences between the asymptotic variances appearing in (2.19) and (2.20). First, we observe the scaling factor $\left(1+H^{-1}\right)$ because the simulation-based estimators have a source of randomness produced by simulation. It is easy to show, however, that this scaling effect is relatively minor for values of $H$ say greater than 3 and below 10. Second, we also notice that $\beta^{p}$ is replaced $b^{p}$, since simulation-based methods rely on a numerical mapping between the $\beta$ vector and $\theta$. A final proposition, stated without proof establishes the relationship between the score-based and Wald SMM estimators.

Proposition 3.3: Under suitable regularity conditions $\hat{\theta}_{N_{p}}^{S S}\left(\Omega_{0}^{-1}\right)$ is asymptotically equivalent to $\hat{\theta}_{N p}^{S W}\left(\Omega_{0}^{-1}\right)$. Further, if $H$ is large, $\hat{\theta}_{T H p}^{S S}\left(S_{T}\right)$ has the same limiting distribution as $\hat{\theta}_{N p}^{S S}\left(S_{T}\right)$, using usual central limit theorem arguments.

\section{Finite-sample performance of estimators: Monte Carlo evidence}

The discussion in Section 2 yielded estimators which represent two key modifications in comparison to the early Durbin method and the more recently proposed procedure by Galbraith and Zinde-Walsh (1994), namely 
the SMM and ALS estimators for $\theta$ both involve a set of overidentified moment conditions and the SMM estimators rely on simulations of the MA process unlike the previously proposed procedures. The six new estimators $\theta_{j}^{i}$ of $\theta$, with $i=S W, S S$ and $A ; j=T H_{p}, N_{p}$ (for $S W$ and $S S$ ) and $j=p$ (for $i=A$ ) have known asymptotic distributions discussed in the previous section. According to those (first-order) asymptotic results we know that the ALS estimators are more efficient than the SMM estimator. Yet, in small samples it was noted by Gouriéroux et al. (1993) that the simulationbased estimator appeared to outperform the asymptotically most efficient ML estimator. We now turn our attention to a Monte Carlo investigation to further explore these small sample properties. All simulations were performed with Gauss Version 3.0 using 1000 replications. All results appear in Talbe 3.1.

We first consider a comparison of $\hat{\theta}_{G / S W}$ and the estimator based on the ALS principle, i.e. $\hat{\theta}_{p}^{A}$. Hence we study first the effect of using overidentifying restrictions to estimate $\theta$ from the $p$-dimensional AR parameter vector. We considered cases of $p=8$ and 12 with sample sizes equal to $T=50$ and 200. These settings correspond to those examined by Galbraith and Zinde-Walsh and were used for comparison. Table 3.1 reports the Bias as well as the Root Mean Squared Error (RMSE). Four values of $\theta$ were considered namely, $\theta=.10, .50, .90, .99$. We continued to use these values for all other experiments as well (this means they differ from the small Monte Carlo investigation in Gouriéroux et al. (1993) who consider $T=250$, and $H=1$ and $p=3)^{5}$.

For low values of $\theta$ we find results of the relative performance of the $\hat{\theta}_{G / S W}, \hat{\theta}_{p}^{A}$ and simulation based estimators as well as the ML estimator that are in line with the asymptotic efficiency rankings. As there is little dependence this is not surprising particularly with $T=200$. For $\theta$ at .5 this ranking is no longer upheld. For instance the ALS generalization of $G / Z W$ with the identity matrix outperforms the original one, but the simulation-based estimators still lag behind.

As $\theta$ approaches the noninvertibility region, like for instance $\theta=.99$, we observe how the simulation based estimators outperform the more traditional estimators. For instance the RMSE of $\hat{\theta}_{G / S W}$ is .1296 for $T=200$ and $p=8$ while for $\hat{\theta}_{N p}^{S W}$ it is .836 with a $50 \%$ reduction in bias. The score-based estimator does not seem to do as well, however as its RMSE is .1479. Also the bias is greatly improved by using the $S W$ type estimator. Since the score-based simulation estimator performed relatively poorly we considered improving it by increasing $H$ from $H=1$ to $H=3$. The results

\footnotetext{
${ }^{5}$ For the sake of space we only report results with $p=8$, results with $p=12$ are available upon request.
} 
in Table 3.1 show however that this had little effect on the performance of the estimator. To summarize, we can say that the simulation based estimators appear to be quite attractive, as they are simple and exhibit desirable small sample properties improving existing methods. Among the simulation based estimators the one based on the Wald principle seems to be the best.

\section{Some extensions}

We would like to conclude by suggesting some relatively straight forward extensions of the estimators proposed in section 2. First and foremost, it is relatively straightforward to extend the SMM estimators to higher order MA models. This can be done without any major modification. Second, we can also consider multivariate MA models. Such extensions are a bit more involved, but conceptually fairly straightforward. 


\section{References}

Ansley, C.F. (1979): “An algorithm for the exact likelihood of a mixed autoregressive moving average process", Biometrika 66, 59-65.

Ansley, C.F. and Newbold, P. (1980): "Finite sample properties of estimators for autoregressive moving average models", Journal of Econometrics $13,159-85$.

Box, G.E.P. and Jenkins G.M. (1976): "Time Series Analysis: Forecasting and Control. (Revised ed.) Holden-Day, San Francisco.

Galbraith J.W. and Zinde-Walsh V. (1994): “A simple, non-iterative estimator for Moving average models", Biometrika 81(1), 143-156.

Gallant A.R. and Tauchen G. (1993): "Which moments to match", Econometric Theory (forthcoming).

Gouriéroux C., Monfort A. and Renault E. (1993): "Indirect inference", Journal of Applied Econometrics 8, S85-S118.

Gouriéroux C., Monfort A. and Trognon (1985): “Moindres Carrés Asymptotiques", Annales de l'INSEE 58, 91-122.

Fuller, W.A. (1976): Introduction to Statistical Time Series, Wiley, New York.

Godolphin, E.J. (1977): “A direct representation for the maximum likelihood estimation of the parameters of a Gaussian moving average process", Biometrika 64, 375-84.

Hannan, E.J. and Rissanen, J. (1982): "Recursive estimation of mixed autoregressive moving average order", Biometrika 69, 81-94.

Koreisha S. and Pukkila T. (1990): "A generalized least squares approach for estimation of autoregressive moving average models", Journal of Time Series Analysis 11, 139-151.

McClave E.J. (1973): "On the bias of AR approximation to Moving Averages", Biometrika 60, 599-605.

Mentz R.P. (1977): "Estimation in the first order Moving Average model through the finite autoregressive approximation: Some asymptotic results", Journal of Econometrics 6, 225-236.

Osborn D.R. (1977): "Exact and approximate maximum likelihood estimators for vector moving average processes", Journal of the Royal Statistical Society, B, 39, 144-118. 
Table 3.1 Bias and RISE of Galbraith and Znde-Walsh, Simulated Method of Moments and

Asymptotic Least Squares Estimators

\begin{tabular}{|c|c|c|c|c|c|c|c|c|c|c|c|c|c|}
\hline \multirow[t]{2}{*}{$T$} & \multirow[t]{2}{*}{$\theta$} & \multicolumn{2}{|c|}{$\hat{\theta}_{G / Z W}$} & \multicolumn{2}{|c|}{$\hat{\theta}_{p}^{A}$ with Identity } & \multicolumn{2}{|c|}{$\hat{\theta}_{p}^{A}$ with $\hat{\Omega}$} & \multirow{2}{*}{$\begin{array}{c}\hat{\theta}_{\text {Thp }}^{S W} \text { or } \\
\text { RMSE }\end{array}$} & \multicolumn{2}{|c|}{$\hat{\theta}_{N p}^{S W}(H=1) \hat{\theta}_{T h p}^{S S}$ or } & \multirow{2}{*}{$\begin{array}{c}\hat{\theta}_{N p}^{S S}(H=1) \\
\text { Bias }\end{array}$} & \multicolumn{2}{|c|}{$\hat{\theta}_{N p}^{S S}(H=3)$} \\
\hline & & RMSE & Bias & RMSE & Bias & RMSE & Bias & & Bias & RMSE & & RMSE & Bias \\
\hline 50 & .10 & .1664 & .0029 & .1940 & .0080 & .2375 & .0068 & .2455 & -.0184 & .2455 & -.0085 & .2451 & -.0122 \\
\hline 200 & .10 & .0730 & -.0004 & .0746 & .0002 & .0845 & .0029 & .1042 & .0002 & .1128 & -.0039 & .1128 & -.0024 \\
\hline 50 & .50 & .1666 & -.0069 & .1525 & -.0040 & .1656 & -.0088 & .2000 & -.0714 & .2379 & -.0857 & .2408 & -.0865 \\
\hline 200 & .50 & .0730 & -.0024 & .0677 & .0002 & .0732 & .0000 & .0874 & -.0111 & .1001 & -.0270 & .1041 & -.0245 \\
\hline 50 & .90 & .1778 & -.0526 & .7250 & .0055 & 1.9179 & .2092 & .2056 & -.0389 & .2409 & -.1814 & 2377 & -.1718 \\
\hline 200 & .90 & .0854 & -.0411 & .0750 & -.0644 & .1266 & -.0623 & .0899 & .0008 & .1019 & -.0702 & .1012 & -.0641 \\
\hline 50 & .99 & 2073 & -.1157 & .3521 & -.1160 & 1.8281 & .0107 & .1681 & -.0442 & .2906 & -.2472 & .2867 & -.2379 \\
\hline 200 & .99 & .1296 & -.1048 & .1384 & -.1326 & 1299 & -.1228 & .0836 & -.0374 & .1488 & -.1296 & .1481 & -1279 \\
\hline
\end{tabular}

Notes: $\hat{\theta}_{G / Z W}$ denotes the estimator proposed by Galbraith and Zinde-Walsh (1994), $\quad \hat{\theta}_{p}^{A} \quad$ is the ALS estimator as defined in (2.16), $\hat{\theta}_{N p}^{S S}$ and $\hat{\theta}_{T H p}^{S W}$ refer to the SMM estimators based on the score principle as defined in (2.14). In the same vein, $\hat{\theta}_{N p}^{S W}$ and $\hat{\theta}_{T H p}^{S W}$ are a notation for the SMM estimators based on the Wald principle as defined in (2.13) and (2.14) 